\title{
Regimes of quantum degeneracy in trapped 1D gases
}

\author{
D.S. Petrov ${ }^{1,2}$, G.V. Shlyapnikov ${ }^{1,2}$, and J.T.M. Walraven ${ }^{1}$ \\ ${ }^{1}$ FOM Institute for Atomic and Molecular Physics, Kruislaan 407, 1098 SJ Amsterdam, The Netherlands \\ ${ }^{2}$ Russian Research Center, Kurchatov Institute, Kurchatov Square, 123182 Moscow, Russia
}

(October 29, 2018)

\begin{abstract}
We discuss the regimes of quantum degeneracy in a trapped 1D gas and obtain the diagram of states. Three regimes have been identified: the BEC regimes of a true condensate and quasicondensate, and the regime of a trapped gas of Tonks (gas of impenetrable bosons). The presence of a sharp cross-over to the BEC regime requires extremely small interaction between particles. We discuss how to distinguish between true and quasicondensates in phase coherence experiments.

03.75.Fi,05.30.Jp
\end{abstract}

Low-temperature 1D Bose systems attract a great deal of interest as they show a remarkable physics not encountered in 2D and 3D. In particular, the 1D Bose gas with repulsive interparticle interaction (the coupling constant $g>0$ ) becomes more non-ideal with decreasing 1D density $n$ [1]. The regime of a weakly interacting gas requires the correlation length $l_{c}=\hbar / \sqrt{m n g}$ ( $m$ is the atom mass) to be much larger than the mean interparticle separation $1 / n$. For small $n$ or large interaction, where this condition is violated, the gas acquires Fermi properties as the wavefunction strongly decreases at short interparticle distances [2, 1]. In this case it is called a gas of impenetrable bosons or gas of Tonks (cf. [3]).

Spatially homogeneous 1D Bose gases with repulsive interparticle interaction have been extensively studied in the last decades. For the delta-functional interaction, Lieb and Liniger [1] have calculated the ground state energy and the spectrum of elementary excitations which at low momenta turns out to be phonon-like. Generalizing the Lieb-Liniger approach, Yang and Yang [⿴囗十 have proved the analyticity of thermodynamical functions at any finite temperature, which indicates the absence of a phase transition. However, at sufficiently low $T$ the correlation properties of a 1D Bose gas are qualitatively different from classical high- $T$ properties. In the regime of a weakly interacting gas $\left(n l_{c} \gg 1\right)$ the density fluctuations are suppressed [5], whereas at finite $T$ the long-wave fluctuations of the phase lead to exponential decay of the one-particle density matrix at large distances [5, 6]. A similar picture, with a power-law decay of the density matrix, was found at $T=0$ [7]:8]. Therefore, the BoseEinstein condensate is absent at any $T$, including $T=0$. Earlier studies of 1D Bose systems are reviewed in [9]. They allow us to conclude that in 1D gases the decrease of temperature leads to a continuous transformation of correlation properties from ideal-gas classical to interaction/statistics dominated. A 1D classical field model for calculating correlation functions in the conditions, where both the density and phase fluctuations are important, was developed in 10 and with respect to Bose gases in [11. Interestingly, $1 \mathrm{D}$ gases can posses the property of superfluidity at $T=0$ [12,9]. Moreover, at finite $T$ one can have metastable supercurrent states which decay on a time scale independent of the size of the system [13].

The earlier discussion of 1D Bose gases was mostly academic as there was no possible realization of such a system. Fast progress in evaporative and optical cooling of trapped atoms and the observation of Bose-Einstein condensation (BEC) in trapped clouds of alkali atoms 114. stimulated a search for non-trivial trapping geometries. At present, there are significant efforts to create (quasi)1D trapped gases 15], where the radial motion of atoms in a cylindrical trap is tightly confined and they only undergo zero point radial oscillations. Then, kinematically the gas is $1 \mathrm{D}$, and the difference from purely 1D gases will only be related to the value of the interparticle interaction which now depends on the radial confinement. The presence of the axial confinement allows one to speak of a trapped 1D gas.

Ketterle and van Druten [16] considered a trapped 1D ideal gas and have revealed an essential role of the discrete structure of the trap levels. They have established that at temperatures $T<N \hbar \omega / \ln 2 N$, where $N$ is the number of particles and $\omega$ the trap frequency, the population of the ground state rapidly grows with decreasing $T$ and becomes macroscopic. Thus, one has a clear BEClike behavior of the ideal trapped $1 \mathrm{D}$ cloud.

A fundamental question concerns the influence of interparticle interaction on the presence and nature of a Bose-condensed state and on the character of a cross-over to the BEC regime. In this Letter we discuss the regimes of quantum degeneracy in a trapped 1D gas with repulsive interparticle interaction. We find that the presence of a sharp cross-over to the BEC regime, predicted in [16], requires extremely small interaction between particles. Otherwise, the decrease of temperature leads to a continuous transformation of a classical gas to quantum degenerate. We identify 3 regimes at $T \ll T_{d}$, where $T_{d} \approx N \hbar \omega$ is the degeneracy temperature. For a sufficiently large interparticle interaction and the number of particles much smaller than a characteristic value $N_{*}$, at any $T \ll T_{d}$ one has a trapped gas of Tonks, with the 
density profile characteristic for an ideal Fermi gas. For $N \gg N_{*}$ we have a weakly interacting gas. The presence of the trapping potential introduces a finite size of the sample and drastically changes the picture of longwave fluctuations of the phase compared to the earlier discussed uniform case. We calculate the density and phase fluctuations and find that well below $T_{d}$ there is a quasicondensate, i.e. the Bose-condensed state where the density fluctuations are suppressed but the phase still fluctuates. At very low $T$ the long-wave fluctuations of the phase are suppressed due to a finite size of the system, and we have a true condensate. The true condensate and the quasicondensate have the same Thomas-Fermi density profile and local correlation properties, and we analyze how to distinguish between these Bose-condensed states in phase coherence experiments.

We first discuss the coupling constant $g$ for possible realizations of $1 \mathrm{D}$ gases. These realizations imply particles in a cylindrical trap, which are tightly confined in the radial $(\rho)$ direction, with the confinement frequency $\omega_{0}$ greatly exceeding the mean-field interaction. Then, at sufficiently low $T$ the radial motion of particles is essentially "frozen" and is governed by the ground-state wavefunction of the radial harmonic oscillator. If the radial extension of the wavefunction, $l_{0}=\left(\hbar / m \omega_{0}\right)^{1 / 2} \gg R_{e}$, where $R_{e}$ is the characteristic radius of the interatomic potential, the interaction between particles acquires a $3 \mathrm{D}$ character and will be characterized by the 3D scattering length $a$. In this case, assuming $l_{0} \gg|a|$, we have

$$
g=2 \hbar^{2} a / m l_{0}^{2} .
$$

This result follows from the analysis in [17] and can also be obtained by averaging the 3D interaction over the radial density profile. Thus, statistical properties of the sample are the same as those of a purely 1D system with the coupling constant given by Eq.(1).

In the regime of a weakly interacting gas, where $n l_{c} \gg$ 1 , we have a small parameter

$$
\gamma=1 /\left(n l_{c}\right)^{2}=m g / \hbar^{2} n \ll 1 .
$$

For particles trapped in a harmonic (axial) potential $V(z)=m \omega^{2} z^{2} / 2$, one can introduce a complementary dimensionless quantity $\alpha=m g l / \hbar^{2}$ which provides a relation between the interaction strength $g$ and the trap frequency $\omega(l=\sqrt{\hbar / m \omega}$ is the amplitude of axial zero point oscillations).

At $T=0$ one has a true condensate: In the ThomasFermi (TF) regime the mean square fluctuations of the phase do not exceed $\sim \gamma^{1 / 2}$ and, hence, these fluctuations are small under the condition (2) (see [18]). The condensate wavefunction is determined by the Gross-Pitaevskii equation (GPE) which gives the well-known TF parabolic density profile $n_{0}(z)=n_{0 \mathrm{~m}}\left(1-z^{2} / R_{T F}^{2}\right)$. The maximum density $n_{0 \mathrm{~m}}=n(0)=\mu / \mathrm{g}$, the TF size of the condensate $R_{T F}=\left(2 \mu / m \omega^{2}\right)^{1 / 2}$, and the chemical potential $\mu=\hbar \omega(3 N \alpha / 4 \sqrt{2})^{2 / 3}$. For $\alpha \gg 1$ we are always in the TF regime $(\mu \gg \hbar \omega)$. In this case, Eq.(2) requires a sufficiently large number of particles:

$$
N \gg N_{*}=\alpha^{2} .
$$

Note that under this condition the ratio $\mu / T_{d} \sim$ $\left(\alpha^{2} / N\right)^{1 / 3} \ll 1$. For $\alpha \ll 1$ the criterion (2) of a weakly interacting gas is satisfied at any $N$, and the condensate is in the TF regime if $N \gg \alpha^{-1}$. In the opposite limit the mean-field interaction is much smaller than the level spacing in the trap $\hbar \omega$. Hence, one has a macroscopic occupation of the ground state of the trap, i.e. there is an ideal gas condensate with a Gaussian density profile.

At this point, we briefly discuss the cross-over to the BEC regime, predicted by Ketterle and van Druten [16. They considered a trapped 1D ideal gas and found that the decrease of temperature to below $T_{c}=N \hbar \omega / \ln 2 N$ leads to a strong increase of the population of the ground state, which rapidly becomes macroscopic. This sharp cross-over originates from the discrete structure of the trap levels and is not observed in quasiclassical calculations 19. We argue that the presence of the interparticle interaction changes the picture drastically. One can only distinguish between the (lowest) trap levels if the interaction between particles occupying a particular level is much smaller than the level spacing. Otherwise the interparticle interaction smears out the discrete structure of the levels. For $T$ close to $T_{c}$ the occupation of the ground state is $\sim T_{c} / \hbar \omega \approx N / \ln 2 N$ [16] and, hence, the mean-field interaction between the particles in this state (per particle) will be $N g / l \ln 2 N$. The sharp BEC crossover requires this quantity to be much smaller than $\hbar \omega$, and we arrive at the condition $N / \ln 2 N \ll \alpha^{-1}$. For a realistic number of trapped particles $\left(N \sim 10^{3}-10^{4}\right)$ this is practically equivalent to the condition at which one has the ideal gas Gaussian condensate (see above).

As we see, the sharp BEC cross-over requires small $\alpha$. For possible realizations of $1 \mathrm{D}$ gases, using the coupling constant $g$ (11), we obtain $\alpha=2 a l / l_{0}^{2}$. Then, even for the ratio $l / l_{0} \sim 10$ and moderate radial confinement with $l_{0} \sim 1 \mu \mathrm{m}$, we have $\alpha \sim 0.1$ for $\mathrm{Rb}$ atoms $(a \approx 50 \AA)$. Clearly, for a reasonably large number of particles the cross-over condition $N \ll \alpha^{-1}$ can only be fulfilled at extremely small interparticle interaction. One can think of reducing $a$ to below $1 \AA$ and achieving $\alpha<10^{-3}$ by using Feshbach resonances as in the MIT and JILA experiments [20]. In this case, already for $N \sim 10^{3}$ one can expect the sharp BEC cross-over and the existence of the Gaussian condensate at $T<T_{c}$.

We now turn to the case of large $\alpha$. For $\gamma \gg 1$ one has a gas of Tonks [1]21]. The one-to-one mapping of this system to a gas of free fermions [2] ensures the fermionic spectrum and density profile of a trapped gas of Tonks. For (axial) harmonic trapping the condition $\gamma \gg 1$ requires $N \ll N_{*}$. The chemi- 
cal potential is equal to $N \hbar \omega$, and the density distribution is $n(z)=n_{m} \sqrt{1-(z / R)^{2}}$, where $n_{m}=\sqrt{2 N} / \pi l$, and the size of the cloud $R=\sqrt{2 N} l$. The density profile $n(z)$ is quite different from both the profile of the zero-temperature condensate and the classical distribution of particles, which provides a root for identifying the trapped gas of Tonks in future experiments. The interference effects and dynamical properties of this system are now a subject of theoretical studies 22,23. In Rb and $\mathrm{Na}$ this regime can be achieved for $N \lesssim 10^{3}$ by the Feshbach increase of $a$ to $\sim 500 \AA$ and using $\omega \sim 1 \mathrm{~Hz}$ and optical radial confinement with $\omega_{0} \sim 10 \mathrm{kHz}(\alpha \sim 50)$.

Large $\alpha$ and $N$ satisfying Eq.(3), or small $\alpha$ and $N \gg$ $\alpha^{-1}$, seem most feasible in experiments with trapped 1D gases. In this case, at any $T \ll T_{d}$ one has a weakly interacting gas in the TF regime. Similarly to the uniform $1 \mathrm{D}$ case, the decrease of temperature to below $T_{d}$ continuously transforms a classical $1 \mathrm{D}$ gas to the regime of quantum degeneracy. At $T=0$ this weakly interacting gas turns to the true TF condensate (see above). It is then subtle to understand how the correlation properties change with temperature at $T \ll T_{d}$. For this purpose, we analyze the behavior of the one-particle density matrix by calculating the fluctuations of the density and phase. We a priori assume small density fluctuations and prove this statement relying on the zero-temperature equations for the mean density $n_{0}(z)$ and excitations. The operator of the density fluctuations is (see, e.g. 25])

$$
\hat{n}^{\prime}(z)=n_{0}^{1 / 2}(z) \sum_{j=1}^{\infty} i f_{j}^{-}(z) \hat{a}_{j}+h . c .
$$

where $\hat{a}_{j}$ is the annihilation operator of the excitation with quantum number $j$ and energy $\epsilon_{j}, f_{j}^{ \pm}=u_{j} \pm v_{j}$, and the $u, v$ functions of the excitations are determined by the same Bogolyubov-de Gennes equations as in the presence of the TF condensate.

The solution of these equations gives the spectrum $\epsilon_{j}=$ $\hbar \omega \sqrt{j(j+1) / 2} 18,24$ and the wavefunctions

$$
f_{j}^{ \pm}(x)=\left(\frac{j+1 / 2}{R_{T F}}\right)^{1 / 2}\left[\frac{2 \mu}{\epsilon_{j}}\left(1-x^{2}\right)\right]^{ \pm 1 / 2} P_{j}(x),
$$

where $j$ is a positive integer, $P_{j}$ are Legendre polynomials, and $x=z / R_{T F}$. For the mean square fluctuations of the density, $\left\langle\left(\delta \hat{n}\left(z, z^{\prime}\right)\right)^{2}\right\rangle=\left\langle\left(\hat{n}^{\prime}(z)-\hat{n}^{\prime}\left(z^{\prime}\right)\right)^{2}\right\rangle$, we have

$$
\frac{\left\langle\left(\delta \hat{n}\left(z, z^{\prime}\right)\right)^{2}\right\rangle}{n_{0 m}^{2}}=\sum_{j=1}^{\infty} \frac{\epsilon_{j}(j+1 / 2)}{2 \mu n_{0 m} R_{T F}}\left(P_{j}(x)-P_{j}\left(x^{\prime}\right)\right)^{2}\left(1+2 N_{j}\right),
$$

with $N_{j}=\left[\exp \left(\epsilon_{j} / T\right)-1\right]^{-1}$ being the occupation numbers for the excitations. At $T \gg \hbar \omega$ the main contribution to the density fluctuations comes from quasiclassical excitations $(j \gg 1)$. The vacuum fluctuations are small: $\left\langle\left(\delta \hat{n}\left(z, z^{\prime}\right)\right)^{2}\right\rangle_{0} \sim n_{0 m}^{2} \gamma^{1 / 2}$. For the thermal fluctuations on a distance scale $\left|z-z^{\prime}\right| \gg l_{c}$, we obtain

$$
\frac{\left\langle\left(\delta n\left(z, z^{\prime}\right)\right)^{2}\right\rangle_{T}}{n_{0 m}^{2}} \approx \frac{T}{T_{d}} \min \left\{\frac{T}{\mu}, 1\right\} .
$$

We see that the density fluctuations are strongly suppressed at temperatures $T \ll T_{d}$. Then, one can write the total field operator as $\hat{\psi}(z)=\sqrt{n_{0}(z)} \exp (i \hat{\phi}(z))$, where $\hat{\phi}(z)$ is the operator of the phase fluctuations, and the one-particle density matrix takes the form (see, e.g. [9])

$$
\left\langle\hat{\psi}^{\dagger}(z) \hat{\psi}\left(z^{\prime}\right)\right\rangle=\sqrt{n_{0}(z) n_{0}\left(z^{\prime}\right)} \exp \left\{-\left\langle\left[\delta \hat{\phi}\left(z, z^{\prime}\right)\right]^{2}\right\rangle / 2\right\}
$$

with $\delta \hat{\phi}\left(z, z^{\prime}\right)=\hat{\phi}(z)-\hat{\phi}\left(z^{\prime}\right)$. The operator of the phase fluctuations is given by (see 25])

$$
\hat{\phi}(z)=\left[4 n_{0}(z)\right]^{-1 / 2} \sum_{j=1}^{\infty} f_{j}^{+}(z) \hat{a}_{j}+h . c .,
$$

and for the mean square fluctuations we have

$$
\left\langle\left[\delta \hat{\phi}\left(z, z^{\prime}\right)\right]^{2}\right\rangle=\sum_{j=1}^{\infty} \frac{\mu(j+1 / 2)}{2 \epsilon_{j} n_{0 m} R_{T F}}\left(P_{j}(x)-P_{j}\left(x^{\prime}\right)\right)^{2}\left(1+2 N_{j}\right) .
$$

For the vacuum fluctuations we find (c.f. [26, 18])

$$
\left\langle\left[\delta \hat{\phi}\left(z, z^{\prime}\right)\right]^{2}\right\rangle_{0} \approx\left(\gamma^{1 / 2} / \pi\right) \ln \left(\left|z-z^{\prime}\right| / l_{c}\right),
$$

and they are small for any realistic size of the gas cloud. The thermal fluctuations of the phase are mostly provided by the contribution of the lowest excitations. A direct calculation, with $N_{j}=T / \epsilon_{j}$, yields

$$
\left\langle\left[\delta \hat{\phi}\left(z, z^{\prime}\right)\right]^{2}\right\rangle_{T}=\frac{4 T \mu}{3 T_{d} \hbar \omega}\left|\log \left[\frac{\left(1-x^{\prime}\right)}{\left(1+x^{\prime}\right)} \frac{(1+x)}{(1-x)}\right]\right| .
$$

In the inner part of the gas sample the logarithm in Eq.(9) is of order unity.

Thus, we can introduce a characteristic temperature

$$
T_{p h}=T_{d} \hbar \omega / \mu
$$

at which the quantity $\left\langle\left[\delta \hat{\phi}\left(z, z^{\prime}\right)\right]^{2}\right\rangle \approx 1$ on a distance scale $\left|z-z^{\prime}\right| \sim R_{T F}$. The characteristic radius of phase fluctuations is $R_{\phi} \approx R_{T F}\left(T_{p h} / T\right) \propto N^{2 / 3} / T$, and for $T<T_{p h}$ it exceeds the sample size $R_{T F}$. This means that at $T \ll T_{p h}$ both the density and phase fluctuations are suppressed, and there is a true condensate. The condition (3) always provides the ratio $T_{p h} / \hbar \omega \approx\left(4 N / \alpha^{2}\right)^{1 / 3} \gg 1$.

In the temperature range, where $T_{d} \gg T \gg T_{p h}$, the density fluctuations are suppressed, but the phase fluctuates on a distance scale $R_{\phi} \ll R_{T F}$. Thus, similarly to the quasi2D case [27, we have a condensate with fluctuating phase (quasicondensate). The radius of the phase fluctuations greatly exceeds the correlation length: $R_{\phi} \approx l_{c}\left(T_{d} / T\right) \gg l_{c}$. Hence, the quasicondensate has the same density profile as the true condensate. Correlation properties at distances smaller than $R_{\phi}$ are also the same. However, the phase fluctuations lead to a drastic difference in the phase coherence properties. 
In Fig.1. we present the state diagram of the trapped $1 \mathrm{D}$ gas for $\alpha=10\left(N_{*}=100\right)$. For $N \gg N_{*}$, the decrease of temperature to below $T_{d}$ leads to the appearance of a quasicondensate which at $T<T_{p h}$ turns to the true condensate. In the $T-N$ plane the approximate border line between the two BEC regimes is determined by the equation $(T / \hbar \omega)=\left(32 N / 9 N_{*}\right)^{1 / 3}$. For $N<N_{*}$ the system can be regarded as a trapped gas of Tonks.

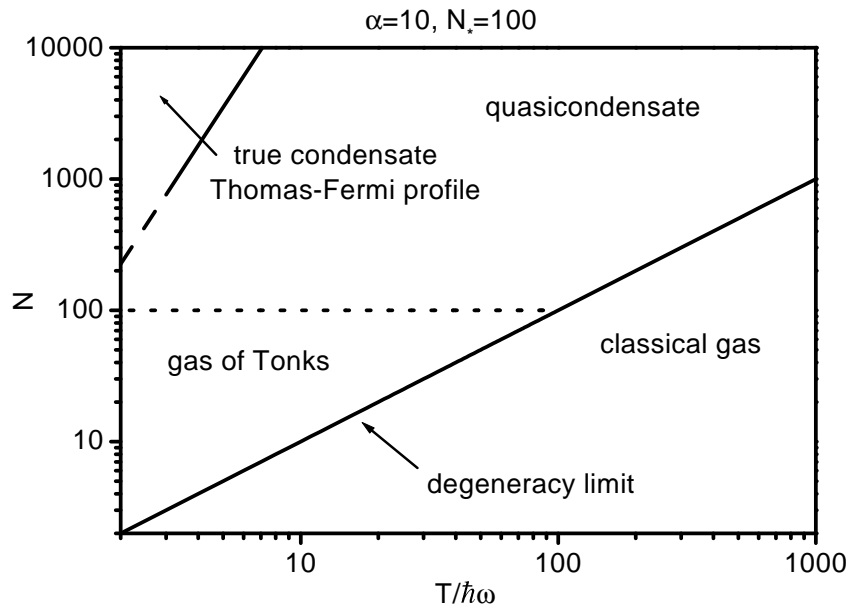

FIG. 1. Diagram of states for a trapped 1D gas.

Phase coherence properties of trapped 1D gases can be studied in 'juggling' experiments similar to those with $3 \mathrm{D}$ condensates at NIST and Munich 28,29. Small clouds of atoms are ejected from the main cloud by stimulated Raman or RF transitions. Observing the interference between two clouds, simultaneously ejected from different parts of the sample, allows the reconstruction of the spatial phase correlation properties. Similarly, temporal correlations of the phase can be studied by overlapping clouds ejected at different times from the same part of the sample. In this way juggling experiments provide a direct measurement of the one-particle density matrix. Repeatedly juggling clouds of a small volume $\Omega$ from points $z$ and $z^{\prime}$ of the sample, for equal time of flight to the detector we have the averaged detection signal $I=\Omega\left[n_{0}(z)+n_{0}\left(z^{\prime}\right)+2\left\langle\hat{\psi}^{\dagger}(z) \psi\left(z^{\prime}\right)\right\rangle\right]$.

At $T \ll T_{p h}$ the phase fluctuations are small and one has a true condensate. In this case, for $z^{\prime}=-z$ we have $\left\langle\hat{\psi}^{\dagger}(z) \hat{\psi}\left(z^{\prime}\right)\right\rangle=n_{0}(z)$ and $I=4 \Omega n_{0}(z)$, and there is a pronounced interference effect: The detected signal is twice as large as the number of atoms in the ejected clouds. The phase fluctuations grow with $T$ and for $T>T_{p h}$, where the true condensate turns to a quasicondensate, the detection signal decreases as described by $\left\langle\hat{\psi}^{\dagger}(z) \hat{\psi}\left(z^{\prime}\right)\right\rangle$ from Eqs. (7) and (9). For $T \gg T_{p h}$ the phase fluctuations completely destroy the interference between the two ejected clouds, and $I=2 \Omega n_{0}(z)$.

In conclusion, we have identified 3 regimes of quantum degeneracy in a trapped $1 \mathrm{D}$ gas: the $\mathrm{BEC}$ regimes of a quasicondensate and true condensate, and the regime of a trapped gas of Tonks. The creation of $1 \mathrm{D}$ gases will open handles on interesting phase coherence studies and the studies of "fermionization" in Bose systems.

This work was financially supported by the Stichting voor Fundamenteel Onderzoek der Materie (FOM), by INTAS, and by the Russian Foundation for Basic Studies.

[1] E.H. Lieb and W. Liniger, Phys. Rev. 130, 1605 (1963); E.H. Lieb, Phys. Rev. 130, 1616 (1963).

[2] M. Girardeau, J. Math. Phys. 1, 516 (1960).

[3] L. Tonks, Phys. Rev. 50, 955 (1936).

[4] C.N. Yang and C.P. Yang, J. Math. Phys. 10, 1115 (1969).

[5] J.W. Kane and L.P. Kadanoff, Phys. Rev. 155, 80 (1967); J. Math. Phys. 6, 1902 (1965).

[6] L. Reatto and G.V. Chester, Phys. Rev. 155, 88 (1967).

[7] M. Schwartz, Phys. Rev. B 15, 1399 (1977).

[8] F.D.M. Haldane, Phys. Rev. Lett. 47, 1840 (1981).

[9] V.N. Popov, Functional Integrals in Quantum Field Theory and Statistical Physics, (D. Reidel Pub., Dordrecht, 1983).

[10] D.J. Scolapino, M. Sears, R.A. Ferrell, Phys. Rev. B 6, 3409 (1972).

[11] Y. Castin et al, J. Mod. Opt., to be published.

[12] E.B. Sonin, Sov. Phys. JETP 32, 773 (1971).

[13] Yu. Kagan, N.V. Prokof'ev and B.V. Svistunov, Phys. Rev. A 61, 045601 (2000).

[14] M.H. Anderson et al, Science 269, 198 (1995); K. Davis et al, Phys. Rev. Lett. 75, 3969 (1995); C.C. Bradley et al, Phys. Rev. Lett. 75, 1687 (1995).

[15] J. Denschlag, D. Cassettari, and J. Schmiedmayer, Phys. Rev. Lett. 82, 2014 (1999); M. Key et al, Phys. Rev. Lett. 84, 1371 (2000).

[16] W. Ketterle and N.J. van Druten, Phys. Rev. A 54, 656 (1996).

[17] M. Olshanii, Phys. Rev. Lett 81, 938 (1998).

[18] Tin-Lun Ho and Michael Ma, J. Low Temp. Phys. 115, 61 (1999).

[19] V. Bagnato and D. Kleppner, Phys. Rev. A 44, 7439 (1991).

[20] J. Stenger et al, Phys. Rev. Lett. 82, 2422 (1999); S.L. Cornish et al, cond-mat/0004290.

[21] The realization of the axially uniform gas of Tonks in a square-well (axial) potential was discussed in 17.

[22] E.B. Kolomeisky et al, cond-mat/0002282,

[23] M.D. Girardeau and E.M. Wright, Phys. Rev. Lett., 84, 5239 (2000).

[24] S. Stringari, Phys. Rev. A 58, 2385 (1998).

[25] S.I. Shevchenko, Sov. J. Low Temp. Phys. 18, 223 (1992).

[26] H. Monien, M. Linn, and N. Elstner, Phys. Rev. A 58, R3395 (1998).

[27] D.S. Petrov, M. Holzmann, and G.V. Shlyapnikov, Phys. Rev. Lett. 84, 2551 (2000).

[28] E. W. Hagley et al, Science 283, 1706 (1999).

[29] I. Bloch et al, Nature 403, 166 (2000). 